\title{
On the Oscillation Criteria for Fourth-Order $p$-Laplacian Differential Equations with Middle Term
}

\author{
Dandan Yang and Chuanzhi Bai \\ Department of Mathematics, Huaiyin Normal University, Huaian, Jiangsu 223300, China \\ Correspondence should be addressed to Chuanzhi Bai; czbai@hytc.edu.cn
}

Received 18 February 2021; Revised 26 February 2021; Accepted 5 April 2021; Published 20 April 2021

Academic Editor: Maria Alessandra Ragusa

Copyright (c) 2021 Dandan Yang and Chuanzhi Bai. This is an open access article distributed under the Creative Commons Attribution License, which permits unrestricted use, distribution, and reproduction in any medium, provided the original work is properly cited.

In this paper, we study the oscillatory properties of the solutions of a class of fourth-order $p$-Laplacian differential equations with middle term. The new oscillation criteria obtained by using the theory of comparison with first- and second-order differential equations and a refinement of the Riccati transformations. The results in this paper improve and generalize the corresponding results in the literatures. Three examples are provided to illustrate our results.

\section{Introduction}

In this paper, we are concerned with the oscillation behavior of solutions of the fourth-order $p$-Laplacian differential equations with middle term

$$
\begin{aligned}
& \left(r(t)\left|z^{\prime \prime \prime}(t)\right|^{p_{1}-2} z^{\prime \prime \prime}(t)\right)^{\prime}+p(t)\left|z^{\prime \prime \prime}(t)\right|^{p_{1}-2} z^{\prime \prime \prime}(t) \\
& \quad+q(t)|x(\sigma(t))|^{p_{2}-2} x(\sigma(t))=0,
\end{aligned}
$$

where $t \geq t_{0}, p_{i}>1 i=1,2$, are real numbers, $z(t):=x(t)+a($ t) $x(\tau(t)), r \in C^{1}\left(\left[t_{0}, \infty\right)\right), p, q, a \in C\left(\left[t_{0}, \infty\right)\right), r(t)>0, q(t)>$ $0, r^{\prime}(t) \geq 0,0 \leq a(t) \leq a_{0}<\infty, \tau \in C^{1}\left[t_{0}, \infty\right), \sigma \in C\left[t_{0}, \infty\right), \tau^{\prime}$ $(t)>0, \tau(t) \leq t$, and $\lim _{t \rightarrow \infty} \tau(t)=\lim _{t \rightarrow \infty} \sigma(t)=\infty$. Furthermore, we investigate (1) under the condition.

$$
\left(H_{1}\right) \lim _{t \rightarrow \infty} \int_{t_{0}}^{t}\left[\frac{1}{r(s)} \exp \left(-\int_{t_{0}}^{s} \frac{p(\xi)}{r(\xi)} d \xi\right)\right]^{1 /\left(p_{1}-1\right)} d s=\infty
$$

A function $z \in C^{\prime \prime \prime}\left[t_{x}, \infty\right), t_{x} \geq t_{0}$, is called a solution of equation (1), if $r(t)\left|z^{\prime \prime \prime}(t)\right|^{p_{1}-2} z^{\prime \prime \prime}(t) \in C^{1}\left(\left[t_{x}, \infty\right)\right.$, and $x$ satisfies (1) on $\left[t_{x}, \infty\right)$.

Since the fourth-order delay differential equations described many real-life applications, such as models related to physical, chemical, and biological phenomena, in the last decades, a lot of research has been done on the oscillatory behavior of fourth-order delay differential equations, see [1-14] and the references cited therein. On the other hand, the $p$-Laplacian differential equations there are some important applications in continuum mechanics and elasticity theory [15-23], the oscillatory behavior of the solutions of fourth-order differential equations with $p$-Laplacian like operator have been investigated in recent years by using different methods and various techniques, for example, [24-26].

In this paper, motivated by [26-28], we will give some new sufficient conditions for that oscillatory behavior of (1). In Section 2, we will provide some lemmas that will help us to prove our main results. In Section 3, based on the comparison with first- and second-order differential equations and a refinement of the Riccati transformations, we establish some new oscillation criteria of (1).

\section{Preliminaries}

First, we give the following lemmas that can discuss our main results.

Lemma 1 ([1], Lemma 2.21). Let $x(t)$ be a positive and $n$ -times differentiable function on an $[T, \infty)$ with its nth derivative $x^{(n)}(t)$ nonpositive on $[T, \infty)$ and not identically zero on 
any interval of the form $\left[T^{\prime}, \infty\right), T^{\prime} \geq T$. Then, there exists an integer $l, 0 \leq l \leq n-1$, with $n+l$ odd such that for some large $T^{*} \geq T$,

$$
\begin{gathered}
(-1)^{l+j} x^{j}>0 \text { on }\left[T^{*}, \infty\right)(j=l, l+1, \cdots, n-1), \\
x^{(i)}>0 \text { on }\left[T^{*}, \infty\right)(i=1,2, \cdots, l-1) \text { when } l>1 .
\end{gathered}
$$

Lemma 2 ([29]). Let the function $x$ satisfies $x^{(i)}(t)>0, i=0$, $1, \cdots, n$, and $x^{(n+1)}(t)<0$ eventually. Then,

$$
\frac{x(t)}{t^{n} / n !} \geq \frac{x^{\prime}(t)}{t^{n-1} /(n-1) !} .
$$

Lemma 3 ([30], Lemma 2.2.3). Let $h \in C^{n}([T, \infty))$ and $h(t)$ $>0$. Assume that $h^{(n)}(t)$ is of a fixed sign, on $\left[t_{0}, \infty\right), h^{(n)}(t)$ not identically zero and that there exists $a t_{1} \geq t_{0}$ such that, for all $t \geq t_{1}, h^{(n-1)}(t) h^{(n)}(t) \leq 0$. If we have $\lim _{t \rightarrow \infty} h(t) \neq 0$, then there exists $t_{\lambda} \geq t_{0}$ such that

$$
h(t) \geq \frac{\lambda}{(n-1) !} t^{n-1}\left|h^{(n-1)}(t)\right|
$$

for every $\lambda \in(0,1)$ and $t \geq t_{\lambda}$.

Lemma 4 ([31], Lemma 2.3). Assume that $\alpha$ is a quotient of odd positive integers; $V>0$ and $U \in \mathbb{R}$ are constants. Then

$$
U y-V y^{(\alpha+1) / \alpha} \leq \frac{\alpha^{\alpha}}{(\alpha+1)^{\alpha+1}} U^{\alpha+1} V^{-\alpha} .
$$

The following lemma will be used in the proof of our main results in the next section.

Lemma 5. Let $x(t)$ be an eventually positive solution of (1); if $\left(H_{1}\right)$ holds, then $z^{\prime}>0$ and $z^{\prime \prime \prime}>0$.

Proof. Since $x(t)$ is an eventually positive solution of (1), then $x(t)>0, x(\tau(t))>0$, and $x(\sigma(t))>0$ for $t \geq t_{1} \geq t_{0}$. Thus, $z($ $t)>0, t \geq t_{1}$. From (1), we obtain

$$
\begin{aligned}
& \left(r(t)\left|z^{\prime \prime \prime}(t)\right|^{p_{1}-2} z^{\prime \prime \prime}(t)\right)^{\prime}+p(t)\left|z^{\prime \prime \prime}(t)\right|^{p_{1}-2} z^{\prime \prime \prime}(t) \\
& \quad=-q(t)|z(\sigma(t))|^{p_{2}-1} z(\sigma(t))<0 .
\end{aligned}
$$

Multiplying by $\exp \left(\int_{t_{0}}^{t}(p(s) / r(s)) d s\right)$ on both sides of the above equation, we get

$$
\begin{aligned}
\frac{d}{d t} & {\left[\exp \left(\int_{t_{0}}^{t} \frac{p(s)}{r(s)} d s\right) r(t)\left|z^{\prime \prime \prime}(t)\right|^{p_{1}-2} z^{\prime \prime \prime}(t)\right] } \\
& =-q(t) \exp \left(\int_{t_{0}}^{t} \frac{p(s)}{r(s)} d s\right)|z(\sigma(t))|^{p_{2}-1} z(\sigma(t))<0 .
\end{aligned}
$$

Thus

$$
\exp \left(\int_{t_{0}}^{t} \frac{p(s)}{r(s)} d s\right) r(t)\left|z^{\prime \prime \prime}(t)\right|^{p_{1}-2} z^{\prime \prime \prime}(t)
$$

is decreasing, and hence, $z^{\prime \prime \prime}(t)$ is eventually of one sign. Hence, we assert that $z^{\prime \prime \prime}(t)>0$ for any $t \geq t_{1}$. Otherwise, if $z^{\prime \prime \prime}(t)<0$ for any $t \geq t_{1}$, we get by (8) that

$$
\begin{aligned}
& \exp \left(\int_{t_{0}}^{t} \frac{p(s)}{r(s)} d s\right) r(t)\left|z^{\prime \prime \prime}(t)\right|^{p_{1}-2} z^{\prime \prime \prime}(t) \\
& \quad \leq \exp \left(\int_{t_{0}}^{t_{1}} \frac{p(s)}{r(s)} d s\right) r\left(t_{1}\right)\left|z^{\prime \prime \prime}\left(t_{1}\right)\right|^{p_{1}-2} z^{\prime \prime \prime}\left(t_{1}\right) \\
& \quad=-M^{p_{1}-1} \exp \left(\int_{t_{0}}^{t_{1}} \frac{p(s)}{r(s)} d s\right),
\end{aligned}
$$

where $M=r\left(t_{1}\right)^{1 / p_{1}-1}\left|z^{\prime \prime \prime}\left(t_{1}\right)\right|>0$. From (10), one has

$$
\left(-z^{\prime \prime \prime}(t)\right)^{p_{1}-1} \geq \frac{M^{p_{1}-1}}{r(t)} \exp \left(-\int_{t_{1}}^{t} \frac{p(s)}{r(s)} d s\right),
$$

that is,

$$
z^{\prime \prime \prime}(t) \leq-M\left[\frac{1}{r(t)} \exp \left(-\int_{t_{1}}^{t} \frac{p(s)}{r(s)} d s\right)\right]^{1 /\left(p_{1}-1\right)} .
$$

Consequently,

$$
\begin{aligned}
z^{\prime \prime}(t) \leq & z^{\prime \prime}\left(t_{1}\right)-M \int_{t_{1}}^{t}\left[\frac{1}{r(s)} \exp \right. \\
& \left.\cdot\left(-\int_{t_{1}}^{s} \frac{p(\xi)}{r(\xi)} d \xi\right)\right]^{1 /\left(p_{1}-1\right)} d s \longrightarrow-\infty \text {, as } t \longrightarrow \infty .
\end{aligned}
$$

So $z(t)$ is an eventually negative function which contradicts $z(t)>0$. Thus, we have $z^{\prime \prime \prime}(t)>0$. From (1), we get

$$
\left(r(t) z^{\prime \prime \prime}(t)^{p_{1}-1}\right)^{\prime}=r^{\prime}(t) z^{\prime \prime \prime}(t)^{p_{1}-1}+\left(p_{1}-1\right) r(t) z^{\prime \prime \prime}(t)^{p_{1}-2} z^{(4)}(t) \leq 0,
$$

from which it follows that

$$
z^{(4)}(t) \leq 0, t \geq t_{1} \geq t_{0}
$$

By (15) and Lemma 1 (set $n=4$ and $l=3$ ), one has that $z^{\prime}(t)>0, t \geq t_{1} \geq t_{0}$. The proof is completed.

Lemma 6 ([32]). Let $\alpha \in(0,1]$ be a quotient of two positive integers. Assume that $R$ is a positive continuous function on $\left(t_{0}, \infty\right)$. If 


$$
\lim _{t \rightarrow \infty} \inf _{\sigma(t)}^{t} R(s) d s>\frac{1}{e}
$$

then the first-order delay differential equation

$$
y^{\prime}(t)+R(t) y^{\alpha}(\sigma(t))=0
$$

is oscillatory.

\section{Main Results}

In the following theorem, we then by using a comparison strategy involving first-order differential equations to provide an oscillation criterion for equation (1).
For convenience, let

$$
A(t)=\frac{1}{a\left(\tau^{-1}(t)\right)}\left(1-\frac{\left(\tau^{-1}\left(\tau^{-1}(t)\right)\right)^{3}}{\left(\tau^{-1}(t)\right)^{3} a\left(\tau^{-1}\left(\tau^{-1}(t)\right)\right)}\right), t \geq t_{0} .
$$

Theorem 7. Assume that $\left(H_{1}\right)$ and

$$
\left(H_{2}\right) \frac{\left(\tau^{-1}\left(\tau^{-1}(t)\right)\right)^{3}}{\left(\tau^{-1}(t)\right)^{3} a\left(\tau^{-1}\left(\tau^{-1}(t)\right)\right)}<1, t \geq t_{0}
$$

hold. If the differential equation

$$
\omega^{\prime}(t)+\left(\frac{\mu}{6}\right)^{p_{2}-1} \frac{q(t) t^{3\left(p_{2}-1\right)} \exp \left(\int_{t_{0}}^{t}(p(s) / r(s)) d s\right) A^{p_{2}-1}(\sigma(t))}{r^{\left(p_{2}-1\right) /\left(p_{1}-1\right)}\left(\tau^{-1}(\sigma(t))\right) \exp \left(\left(p_{2}-1\right) /\left(p_{1}-1\right) \int_{t_{0}}^{\tau^{-1}(\sigma(t))}(p(s) / r(s)) d s\right)} \omega^{\left(p_{2}-1\right) /\left(p_{1}-1\right)}\left(\tau^{-1}(\sigma(t))\right)=0,
$$

is oscillatory for some $\mu \in(0,1)$, then the differential equation (1) is oscillatory.

Proof. Assume that (1) has a nonoscillatory solution in $\left[t_{0}\right.$, $\infty)$. Without loss of generality, we may let $x$ be an eventually positive solution of (1). Then, there exists a $t_{1} \geq t_{0}$ such that $x(t)>0, x(\tau(t))>0$, and $x(\sigma(t))>0$ for $t \geq t_{1}$. Let

$\omega(t):=r(t) \exp \left(\int_{t_{0}}^{t} \frac{p(s)}{r(s)} d s\right) z^{\prime \prime \prime}(t)^{p_{1}-1}>0[$ from Lemma 5],

which having in mind (1) gives

$$
\omega^{\prime}(t)+q(t) \exp \left(\int_{t_{0}}^{t} \frac{p(s)}{r(s)} d s\right) x(\sigma(t))^{p_{2}-1}=0
$$

From the definition of $z(t)$, one has

$$
x(t)=\frac{1}{a\left(\tau^{-1}(t)\right)}\left(z\left(\tau^{-1}(t)\right)-x\left(\tau^{-1}(t)\right)\right) .
$$

By repeating the same process, we have

$$
\begin{aligned}
x(t) & =\frac{z\left(\tau^{-1}(t)\right)}{a\left(\tau^{-1}(t)\right)}-\frac{1}{a\left(\tau^{-1}(t)\right)}\left(\frac{z\left(\tau^{-1}\left(\tau^{-1}(t)\right)\right)}{a\left(\tau^{-1}\left(\tau^{-1}(t)\right)\right)}-\frac{x\left(\tau^{-1}\left(\tau^{-1}(t)\right)\right)}{a\left(\tau^{-1}\left(\tau^{-1}(t)\right)\right)}\right) \\
& \geq \frac{z\left(\tau^{-1}(t)\right)}{a\left(\tau^{-1}(t)\right)}-\frac{1}{a\left(\tau^{-1}(t)\right)} \frac{z\left(\tau^{-1}\left(\tau^{-1}(t)\right)\right)}{a\left(\tau^{-1}\left(\tau^{-1}(t)\right)\right)} .
\end{aligned}
$$

Set $n=3$ in Lemma 2, we obtain $z(t) \geq(1 / 3) t z^{\prime}(t)$, which implies that $z(t) / t^{3}$ is nonincreasing. Moreover, by the fact $\tau(t) \leq t$ that gives

$$
\left(\tau^{-1}(t)\right)^{3} z\left(\tau^{-1}\left(\tau^{-1}(t)\right)\right) \leq\left(\tau^{-1}\left(\tau^{-1}(t)\right)\right)^{3} z\left(\tau^{-1}(t)\right) .
$$

Combining (24) and (25), which yields

$$
\begin{aligned}
x(t) & \geq \frac{1}{a\left(\tau^{-1}(t)\right)}\left(1-\frac{\left(\tau^{-1}\left(\tau^{-1}(t)\right)^{3}\right.}{\left(\tau^{-1}(t)\right)^{3} a\left(\tau^{-1}\left(\tau^{-1}(t)\right)\right)}\right) z\left(\tau^{-1}(t)\right) \\
& =A(t) z\left(\tau^{-1}(t)\right) .
\end{aligned}
$$

Between equations (1) and (26), we obtain

$$
\omega^{\prime}(t)+q(t) \exp \left(\int_{t_{0}}^{t} \frac{p(s)}{r(s)} d s\right) A^{p_{2}-1}(\sigma(t)) z^{p_{2}-1}\left(\tau^{-1}(\sigma(t))\right) \leq 0
$$

Since $z$ is positive and increasing (by Lemma 5), we have $\lim _{t \rightarrow \infty} z(t)=0$. So, from Lemma 3, one has

$$
z(t) \geq \frac{\mu}{6} t^{3} z^{\prime \prime \prime}(t)
$$

for some $\mu \in(0,1)$. It follows between (27) and (28) that, for all $\mu \in(0,1), \omega$ is a positive solution of the first-order delay differential inequality 


$$
\omega^{\prime}(t)+\left(\frac{\mu}{6}\right)^{p_{2}-1} \frac{q(t) t^{3\left(p_{2}-1\right)} \exp \left(\int_{t_{0}}^{t}(p(s) / r(s)) d s\right) A^{p_{2}-1}(\sigma(t))}{r^{\left(p_{2}-1\right) /\left(p_{1}-1\right)}\left(\tau^{-1}(\sigma(t))\right) \exp \left(\left(p_{2}-1\right) /\left(p_{1}-1\right) \int_{t_{0}}^{\tau^{-1}(\sigma(t))}(p(s) / r(s)) d s\right)} \omega^{\left(p_{2}-1\right) /\left(p_{1}-1\right)}\left(\tau^{-1}(\sigma(t))\right) \leq 0
$$

It is well known (see [33] and Theorem 7) that the corresponding equation (20) also has a positive solution, which is

Corollary 8. Assume that $\left(H_{1}\right)$ and $\left(H_{2}\right)$ hold, and $p_{1} \geq p_{2}$. If a contradiction. The theorem is proved.

$$
\lim _{t \rightarrow \infty} \inf _{\tau^{-1}(\sigma(t))}^{t}\left(\frac{\mu}{6}\right)^{p_{2}-1} \frac{q(s) s^{3\left(p_{2}-1\right)} \exp \left(\int_{t_{0}}^{s}(p(\xi) / r(\xi)) d \xi\right) A^{p_{2}-1}(\sigma(s))}{r^{\left(p_{2}-1\right) /\left(p_{1}-1\right)}\left(\tau^{-1}(\sigma(s))\right) \exp \left(\left(p_{2}-1\right) /\left(p_{1}-1\right) \int_{t_{0}}^{\tau^{-1}(\sigma(s))}(p(\xi) / r(\xi)) d \xi\right)} d s>\frac{1}{e}
$$

for some $\mu \in(0,1)$, then the differential equation (1) is oscillatory.

Proof. From Lemma 6, we know that (30) implies the oscillatory of (20).
Theorem 9. Assume that $\left(H_{1}\right)$ and $\left(H_{3}\right) a \in C\left(\left[t_{0}, \infty\right)\right), 0 \leq$ $a(t)<a_{0}<1$ hold. If the differential equation

$$
\omega^{\prime}(t)+\left(\frac{\mu}{6}\right)^{p_{2}-1} \frac{q(t)(\sigma(t))^{3\left(p_{2}-1\right)} \exp \left(\int_{t_{0}}^{t}(p(s) / r(s)) d s\right)(1-a(\sigma(t)))^{p_{2}-1}}{r^{\left(p_{2}-1\right) /\left(p_{1}-1\right)}(\sigma(t)) \exp \left(\left(p_{2}-1\right) /\left(p_{1}-1\right) \int_{t_{0}}^{\sigma(t)}(p(s) / r(s)) d s\right)} \omega^{\left(p_{2}-1\right) /\left(p_{1}-1\right)}(\sigma(t))=0,
$$

is oscillatory, then every solution of equation (1) is oscillatory.

Proof. Assume the contrary that $x$ is an eventually positive solution of equation (1). Thus, we may suppose that $x(t), x$ $(\tau(t))$, and $x(\sigma(t))$ are positive for all $t \geq t_{1}$ that are sufficiently large. From $z(t)=x(t)+a(t) x(\tau(t))$ and $z^{\prime}(t)>0$ (by Lemma 5), we obtain by $x(t) \leq z(t)$ and $\tau(t) \leq t$ that

$$
\begin{aligned}
x(t) & =z(t)-a(t) x(\tau(t)) \geq z(t)-a(t) z(\tau(t)) \\
& \geq(1-a(t)) z(t) .
\end{aligned}
$$

Combining (21) and (32), one has

$$
\omega^{\prime}(t)+q(t) \exp \left(\int_{t_{0}}^{t} \frac{p(s)}{r(s)} d s\right)(1-a(\sigma(t)))^{p_{2}-1} z(\sigma(t))^{p_{2}-1} \leq 0
$$

By using (28) and (33), we get that

$$
\begin{aligned}
\omega^{\prime}(t)+ & \left(\frac{\mu}{6}\right)^{p_{2}-1} q(t)(\sigma(t))^{3\left(p_{2}-1\right)} \exp \\
& \cdot\left(\int_{t_{0}}^{t} \frac{p(s)}{r(s)} d s\right)(1-a(\sigma(t)))^{p_{2}-1}\left(z^{\prime \prime \prime}(\sigma(t))\right)^{p_{2}-1} \leq 0 .
\end{aligned}
$$

From (21), (34) implies that

$$
\omega^{\prime}(t)+\left(\frac{\mu}{6}\right)^{p_{2}-1} \frac{q(t)(\sigma(t))^{3\left(p_{2}-1\right)} \exp \left(\int_{t_{0}}^{t}(p(s) / r(s)) d s\right)(1-a(\sigma(t)))^{p_{2}-1}}{r^{\left(p_{2}-1\right) /\left(p_{1}-1\right)}(\sigma(t)) \exp \left(\left(p_{2}-1\right) /\left(p_{1}-1\right) \int_{t_{0}}^{\sigma(t)}(p(s) / r(s)) d s\right)} \omega^{\left(p_{2}-1\right) /\left(p_{1}-1\right)}(\sigma(t)) \leq 0 .
$$


It is well known (see [33] and Theorem 7) that the corresponding equation (31) also has a positive solution, which is a contradiction. The theorem is proved.
Corollary 10. Assume that $\left(H_{1}\right)$ and $\left(H_{3}\right)$ hold, and $p_{1} \geq p_{2}$. If

$$
\lim _{t \rightarrow \infty} \inf _{\sigma(t)}^{t}\left(\frac{\mu}{6}\right)^{p_{2}-1} \frac{q(s)(\sigma(s))^{3\left(p_{2}-1\right)} \exp \left(\int_{t_{0}}^{s} p(\xi) / r(\xi) d \xi\right)(1-a(\sigma(s)))^{p_{2}-1}}{r^{\left(p_{2}-1\right) /\left(p_{1}-1\right)}(\sigma(s)) \exp \left(\left(p_{2}-1\right) /\left(p_{1}-1\right) \int_{t_{0}}^{\sigma(s)}(p(\xi) / r(\xi)) d \xi\right)} d s>\frac{1}{e}
$$

for some $\mu \in(0,1)$, then the differential equation (1) is oscillatory.

Proof. From Lemma 6, we know that (36) implies the oscillatory of (31).

Lemma 11. Assume that $\left(H_{1}\right)$ holds, $x$ is an eventually positive solution of (1), and

$$
\begin{gathered}
\int_{t_{2}}^{\infty}\left(h(s) q(s) \exp \left(\int_{t_{0}}^{s} \frac{p(\xi)}{r(\xi)} d \xi\right) \frac{\sigma(s)^{3\left(p_{1}-1\right)}}{s^{3\left(p_{1}-1\right)}}\right. \\
\left.-\frac{2^{p_{1}-1}}{p_{1}^{p_{1}}} \frac{r(s)\left(h^{\prime}(s)\right)^{p_{1}}}{\left(\varepsilon_{1} s^{2} h(s)\right)^{p_{1}-1} \exp \left(-\int_{t_{0}}^{s}(p(\xi) / r(\xi)) d \xi\right)}\right) d s=\infty,
\end{gathered}
$$

for some constants $\varepsilon_{1} \in(0,1)$. Then, we have $z^{\prime \prime}<0$.

Proof. Our proof by reduction to the absurd. Assume that $z^{\prime \prime}(t)>0$. From Lemma 2, we obtain

$$
\frac{z^{\prime}(t)}{z(t)} \leq \frac{3}{t}
$$

Integrating the above equality from $\sigma(t)$ to $t$, one find that

$$
\frac{z(\sigma(t))}{z(t)} \geq \frac{(\sigma(t))^{3}}{t^{3}}
$$

Let $h(t)=z^{\prime}(t)$ in Lemma 3 , then

$$
z^{\prime}(t) \geq \frac{\varepsilon_{1}}{2} t^{2} z^{\prime \prime \prime}(t)
$$

for all $\varepsilon_{1} \in(0,1)$ and every sufficiently large $t$. Now, we define a function $\phi$ by

$$
\phi(t):=h(t) \frac{\exp \left(\int_{t_{0}}^{t}(p(s) / r(s)) d s\right) r(t)\left(z^{\prime \prime \prime}(t)\right)^{p_{1}-1}}{z^{p_{1}-1}(t)}>0 .
$$

By differentiating (41) and using the inequalities (39) and (40), we get

$$
\begin{aligned}
\phi^{\prime}(t) \leq & \frac{h^{\prime}(t)}{h(t)} \phi(t)-h(t) q(t) \exp \\
& \cdot\left(\int_{t_{0}}^{t} \frac{p(s)}{r(s)} d s\right) \frac{\sigma(t)^{3\left(p_{1}-1\right)}}{t^{3\left(p_{1}-1\right)^{6}}} z^{p_{2}-p_{1}}(\sigma(t)) \\
& -\frac{\left(p_{1}-1\right) \varepsilon_{1}}{2} \frac{t^{2} \exp \left(-1 /\left(p_{1}-1\right) \int_{t_{0}}^{t}(p(s) / r(s)) d s\right)}{h^{1 /\left(p_{1}-1\right)}(t) r^{1 /\left(p_{1}-1\right)}(t)} \phi^{1+1 /\left(p_{1}-1\right)} .
\end{aligned}
$$

Since $z^{\prime}(t)>0$, there exist a $t_{2} \geq t_{1}$ and a constant $M>0$ such that $z(t)>M$, for all $t \geq t_{2}$. Without loss of generality, we may let $M \geq 1$. By using Lemma 4 with

$$
\begin{aligned}
& U=\frac{h^{\prime}}{h}, \\
& V=\frac{\left(p_{1}-1\right) \varepsilon_{1}}{2} \frac{t^{2} \exp \left(-\left(1 /\left(p_{1}-1\right)\right) \int_{t_{0}}^{t}(p(s) / r(s)) d s\right)}{h^{1 /\left(p_{1}-1\right)}(t) r^{1 /\left(p_{1}-1\right)}(t)},
\end{aligned}
$$

we obtain

$$
\begin{aligned}
\phi^{\prime}(t) \leq & -M^{p_{2}-p_{1}} h(t) q(t) \exp \left(\int_{t_{0}}^{t} \frac{p(s)}{r(s)} d s\right) \frac{\sigma(t)^{3\left(p_{1}-1\right)}}{t^{3\left(p_{1}-1\right)}} \\
& +\frac{2^{p_{1}-1}}{p_{1}^{p_{1}}} \frac{r(t)\left(h^{\prime}(t)\right)^{p_{1}}}{\left(\varepsilon_{1} t^{2} h(t)\right)^{p_{1}-1} \exp \left(-\int_{t_{0}}^{t}(p(s) / r(s)) d s\right)} .
\end{aligned}
$$


This implies that

$$
\begin{gathered}
\int_{t_{2}}^{t}\left(h(s) q(s) \exp \left(\int_{t_{0}}^{s} \frac{p(\xi)}{r(\xi)} d \xi\right) \frac{\sigma(s)^{3\left(p_{1}-1\right)}}{s^{3\left(p_{1}-1\right)}}\right. \\
\left.-\frac{2^{p_{1}-1}}{p_{1}^{p_{1}}} \frac{r(s)\left(h^{\prime}(s)\right)^{p_{1}}}{\left(\varepsilon_{1} s^{2} h(s)\right)^{p_{1}-1} \exp \left(-\int_{t_{0}}^{s}(p(\xi) / r(\xi)) d \xi\right)}\right) d s \leq \phi\left(t_{2}\right), \\
\leq \int_{t_{2}}^{t}\left(M^{p_{2}-p_{1}} h(s) q(s) \exp \left(\int_{t_{0}}^{s} \frac{p(\xi)}{r(\xi)} d \xi\right) \frac{\sigma(s)^{3\left(p_{1}-1\right)}}{s^{3\left(p_{1}-1\right)}}\right. \\
\left.-\frac{2^{p_{1}-1}}{p_{1}^{p_{1}}} \frac{r(s)\left(h^{\prime}(s)\right)^{p_{1}}}{\left(\varepsilon_{1} s^{2} h(s)\right)^{p_{1}-1} \exp \left(-\int_{t_{0}}^{s}(p(\xi) / r(\xi)) d \xi\right)}\right) d s \leq \phi\left(t_{2}\right),
\end{gathered}
$$

which contradicts (37). The proof is completed.

For convenience, let

$$
\begin{aligned}
\theta(t)= & \lambda^{\left(p_{2}-1\right) /\left(p_{1}-1\right)} \int_{t}^{\infty}\left(\frac{1}{r(\xi)} \int_{\xi}^{\infty} q(s) \exp \right. \\
& \left.\cdot\left(\int_{t}^{s} \frac{p(\xi)}{r(\xi)} d \xi\right)\left(\frac{\sigma(s)}{s}\right)^{p_{2}-1} d s\right)^{1 /\left(p_{1}-1\right)} d \xi
\end{aligned}
$$

Theorem 12. Assume that $\left(H_{1}\right)$ and (37) hold for some $\varepsilon_{1} \in$ $(0,1)$ and $p_{2} \geq p_{1}$. If

$$
y^{\prime \prime}(t)+\theta(t) y(t)=0
$$

is oscillatory, then (1) is also oscillatory.

Proof. We use the reduction to the absurd arguments. Assume that (1) has a nonoscillatory solution in $\left[t_{0}, \infty\right)$. Without loss of generality, we only need to be concerned with positive solutions of equation (1). Then, there exists a $t_{1} \geq t_{0}$ such that $x(t)>0, x(\tau(t))>0$, and $x(\sigma(t))>0$ for $t \geq t_{1}$. From Lemmas 4 and 11, one has that

$$
\begin{aligned}
& z^{\prime}(t)>0, \\
& z^{\prime \prime}(t)<0, \\
& z^{\prime \prime \prime}(t)>0,
\end{aligned}
$$

for $t \geq t_{2}$, where $t_{2}$ is sufficiently large. Integrating (8) from $t$ to $\rho$, we obtain

$$
\begin{aligned}
\exp & \left(\int_{t_{0}}^{\rho} \frac{p(s)}{r(s)} d s\right) r(\rho)\left(z^{\prime \prime \prime}(\rho)\right)^{p_{1}-1} \\
= & \exp \left(\int_{t_{0}}^{t} \frac{p(s)}{r(s)} d s\right) r(t)\left(z^{\prime \prime \prime}(t)\right)^{p_{1}-1} \\
& -\int_{t}^{\rho} q(s) \exp \left(\int_{t_{0}}^{s} \frac{p(\xi)}{r(\xi)} d \xi\right) z^{p_{2}-1}(\sigma(s)) d s .
\end{aligned}
$$

By using Lemma 3 in [26] together with (48), we have

$$
\frac{z(\sigma(t))}{z(t)} \geq \lambda \frac{\sigma(t)}{t}
$$

for all $\lambda \in(0,1)$. This coupled with (49); we can arrive at

$$
\begin{aligned}
& \exp \left(\int_{t_{0}}^{\rho} \frac{p(s)}{r(s)} d s\right) r(\rho)\left(z^{\prime \prime \prime}(\rho)\right)^{p_{1}-1} \\
& \quad-\exp \left(\int_{t_{0}}^{t} \frac{p(s)}{r(s)} d s\right) r(t)\left(z^{\prime \prime \prime}(t)\right)^{p_{1}-1} \\
& +\lambda^{p_{2}-1} \int_{t}^{\rho} q(s) \exp \left(\int_{t_{0}}^{s} \frac{p(\xi)}{r(\xi)} d \xi\right)\left(\frac{\sigma(s)}{s}\right)^{p_{2}-1} z^{p_{2}-1}(s) d s \leq 0 .
\end{aligned}
$$

Since $z^{\prime}>0$, then (51) reduced to the following inequality

$$
\begin{aligned}
& \exp \left(\int_{t_{0}}^{\rho} \frac{p(s)}{r(s)} d s\right) r(\rho)\left(z^{\prime \prime \prime}(\rho)\right)^{p_{1}-1} \\
& \quad-\exp \left(\int_{t_{0}}^{t} \frac{p(s)}{r(s)} d s\right) r(t)\left(z^{\prime \prime \prime}(t)\right)^{p_{1}-1} \\
& +\lambda^{p_{2}-1} z^{p_{2}-1}(t) \int_{t}^{\rho} q(s) \exp \left(\int_{t_{0}}^{s} \frac{p(\xi)}{r(\xi)} d \xi\right)\left(\frac{\sigma(s)}{s}\right)^{p_{2}-1} d s \leq 0 .
\end{aligned}
$$

Taking $\rho \longrightarrow \infty$ in (52), one has

$$
\begin{aligned}
& -\exp \left(\int_{t_{0}}^{t} \frac{p(s)}{r(s)} d s\right) r(t)\left(z^{\prime \prime \prime}(t)\right)^{p_{1}-1} \\
& +\lambda^{p_{2}-1} z^{p_{2}-1}(t) \int_{t}^{\infty} q(s) \exp \left(\int_{t_{0}}^{s} \frac{p(\xi)}{r(\xi)} d \xi\right)\left(\frac{\sigma(s)}{s}\right)^{p_{2}-1} d s \leq 0,
\end{aligned}
$$

from which we readily obtain

$$
\begin{aligned}
z^{\prime \prime \prime}(t) \geq & \frac{\lambda^{\left(p_{2}-1\right) /\left(p_{1}-1\right)}}{r^{1 /\left(p_{1}-1\right)}(t)} \exp \left(-\frac{1}{p_{1}-1} \int_{t_{0}}^{t} \frac{p(s)}{r(s)} d s\right) z^{\left(p_{2}-1\right) /\left(p_{1}-1\right)}(t) \\
& \times\left(\int_{t}^{\infty} q(s) \exp \left(\int_{t_{0}}^{s} \frac{p(\xi)}{r(\xi)} d \xi\right)\left(\frac{\sigma(s)}{s}\right)^{p_{2}-1} d s\right)^{1 /\left(p_{1}-1\right)} .
\end{aligned}
$$

Integrating the above inequality from $t$ to $\infty$, we obtain

$$
\begin{aligned}
-z^{\prime \prime}(t) \geq & \lambda^{\left(p_{2}-1\right) /\left(p_{1}-1\right)} z^{\left(p_{2}-1\right) /\left(p_{1}-1\right)}(t) \\
& \times \int_{t}^{\infty}\left(\frac{1}{r(\xi)} \int_{\xi}^{\infty} q(s) \exp \left(\int_{t}^{s} \frac{p(\xi)}{r(\xi)} d \xi\right)\right. \\
& \left.\cdot\left(\frac{\sigma(s)}{s}\right)^{p_{2}-1} d s\right)^{1 /\left(p_{1}-1\right)} d \xi
\end{aligned}
$$


which implies that

$$
z^{\prime \prime}(t) \leq-\theta(t) z^{\left(p_{2}-1\right) /\left(p_{1}-1\right)}(t) .
$$

Now, if we define $\gamma(t)=z^{\prime}(t) / z(t)$, then $\gamma(t)>0$ for $t$ $\geq t_{1}$, and

$$
\gamma^{\prime}(t)=\frac{z^{\prime \prime}(t)}{z(t)}-\left(\frac{z^{\prime}(t)}{z(t)}\right)^{2} .
$$

By using (56) and the definition of $\gamma(t)$, we see that

$$
\gamma^{\prime}(t) \leq-\theta(t) \frac{z^{\left(p_{2}-1\right) /\left(p_{1}-1\right)}(t)}{z(t)}-\gamma^{2}(t) .
$$

Since $z^{\prime}(t)>0$, there exists a constant $M>0$ such that $z(t) \geq M^{p_{2}-1}$, for all $t \geq t_{2}$, where $t_{2}$ is sufficiently large. Without loss of generality, we may let $M \geq 1$. Then, by (56), one has

$$
\gamma^{\prime}(t)+\gamma^{2}(t)+\theta(t) \leq \gamma^{\prime}(t)+\gamma^{2}(t)+M^{p_{2}-p_{1}} \theta(t) \leq 0 .
$$

It is well known (see [34]) that the differential equation (47) is oscillatory if and only if there exists a $t_{3}>\max \left\{t_{1}\right.$, $t_{2}$ \} such that (59) holds, which is a contradiction. The theorem is proved.

For convenience, let

$$
\beta(t)=\int_{t}^{\infty}\left(\frac{1}{r(\xi)} \int_{\xi}^{\infty} q(s) \exp \left(\int_{t}^{s} \frac{p(\xi)}{r(\xi)} d \xi\right) d s\right)^{1 /\left(p_{1}-1\right)} d \xi
$$

Theorem 13. Assume that $\left(H_{1}\right)$ and (37) hold for some $\varepsilon_{1} \in$ $(0,1), p_{2} \geq p_{1}$, and $\sigma^{\prime}(t) \geq 1$. If

$$
\left(\frac{1}{\sigma^{\prime}(t)} y^{\prime}(t)\right)^{\prime}+\beta(t) y(t)=0,
$$

is oscillatory, then (1) is also oscillatory.

Proof. As in the proof of Theorem 12, one has (49). Hence, it follows between $\sigma^{\prime}(t) \geq 0$ and $z^{\prime}(t) \geq 0$ that

$$
\begin{aligned}
& \exp \left(\int_{t_{0}}^{\rho} \frac{p(s)}{r(s)} d s\right) r(\rho)\left(z^{\prime \prime \prime}(\rho)\right)^{p_{1}-1} \\
& -\exp \left(\int_{t_{0}}^{t} \frac{p(s)}{r(s)} d s\right) r(t)\left(z^{\prime \prime \prime}(t)\right)^{p_{1}-1} \\
& +z^{p_{2}-1}(\sigma(t)) \int_{t}^{\rho} q(s) \exp \left(\int_{t}^{s} \frac{p(\xi)}{r(\xi)} d \xi\right) d s \leq 0 .
\end{aligned}
$$

By (62), similar to (56), we obtain

$$
z^{\prime \prime}(t) \leq-\beta(t) z^{\left(p_{2}-1\right) /\left(p_{1}-1\right)}(\sigma(t)) .
$$

We now define $\kappa$ by

$$
\kappa(t)=\frac{z^{\prime}(t)}{z(\sigma(t))},
$$

then $\kappa(t)>0$ for $t \geq t_{1}$, and

$$
\begin{aligned}
\kappa^{\prime}(t) & =\frac{z^{\prime \prime}(t)}{z(\sigma(t))}-\frac{z^{\prime}(t)}{z^{2}(\sigma(t))} z^{\prime}(\sigma(t)) \sigma^{\prime}(t) \\
& \leq \frac{z^{\prime \prime}(t)}{z(\sigma(t))}-\left(\frac{z^{\prime}(t)}{z(\sigma(t))}\right)^{2} \sigma^{\prime}(t) .
\end{aligned}
$$

By using (63) and the definition of $\kappa(t)$, similar to (59), we see that

$$
\kappa^{\prime}(t)+\beta(t)+\sigma^{\prime}(t) \kappa^{2}(t) \leq 0 .
$$

It is well known (see [34]) that the differential equation in (61) is nonoscillatory if and only if there exists a $t_{3}>\max \{$ $\left.t_{1}, t_{2}\right\}$ such that (66) holds, which is a contradiction. So, the theorem is proved.

In the following, we employ the integral averaging technique to establish a Philos-type oscillation criterion for (1).

Let

$$
\begin{aligned}
D & =\left\{(t, s) \in R^{2}: t \geq s \geq t_{0}\right\}, \\
D_{0} & =\left\{(t, s) \in R^{2}: t>s \geq t_{0}\right\} .
\end{aligned}
$$

Corollary 14. Assume that $\left(H_{1}\right)$ and (37) hold for some $\varepsilon_{1} \epsilon$ $(0,1)$ and $p_{1}=p_{2}$. Let there exists a continuous function $H$ $: D \longrightarrow R$ such that $H(t, s)=0$ for $t \geq t_{0}, H(t, s)>0,(t, s) \in$ $D_{0}$, and $H$ has a nonpositive continuous partial derivative with respect to the second variable in $D$. Suppose that there exists function $h \in C(D, R)$ such that

$$
-\frac{\partial}{\partial s} H(t, s)=h(t, s) \sqrt{H(t, s)} \text { for all }(t, s) \in D .
$$

$$
\text { If }
$$

$$
\lim _{t \rightarrow \infty} \frac{1}{H\left(t, t_{0}\right)} \int_{t_{0}}^{t}\left[H(t, s) \theta(s)-\frac{1}{4} h^{2}(t, s)\right] d s=\infty,
$$

or

$$
\lim _{t \rightarrow \infty} \inf _{t} t \int_{t}^{\infty} \theta(s) d s>\frac{1}{4},
$$

then (1) is oscillatory. 
Example 15. Consider the equation

$$
z^{(4)}(t)+\frac{1}{t} z^{\prime \prime \prime}(t)+\frac{q_{0}}{t^{4}} x\left(\sigma_{0} t\right)=0
$$

where $t \geq 1, z(t)=x(t)+a_{0} x\left(\tau_{0} t\right), q_{0}>0, \tau_{0} \in\left(a_{0}^{-1 / 3}, 1\right)$, and $\sigma_{0} \in\left(0, \tau_{0}\right)$. Let $p_{1}=p_{2}=2, r(t)=1, a(t)=a_{0}, \tau(t)=\tau_{0} t, \sigma$ $(t)=\sigma_{0} t, p(t)=1 / t$, and $q(t)=q_{0} / t^{4}$. It is easy to check that $\left(H_{1}\right)$ and $\left(H_{2}\right)$ are satisfied. Moreover, we have

$$
A(t)=\frac{1}{a_{0}}\left(1-\frac{1}{a_{0} \tau_{0}^{3}}\right) .
$$

If $q_{0}>0$ satisfies inequality (30), that is

$$
\begin{aligned}
& \lim _{t \rightarrow \infty} \inf _{\sigma_{0} / \tau_{0} t}^{t} \frac{\mu}{6} \frac{q_{0}}{s^{4}} s^{3} A\left(\sigma_{0} s\right) \exp \left(\int_{\sigma_{0} / \tau_{0} s}^{s} \frac{1}{\xi} d \xi\right) d s \\
& \quad=q_{0} \frac{\mu}{6} \frac{1}{a_{0}}\left(1-\frac{1}{a_{0} \tau_{0}^{3}}\right) \frac{\tau_{0}}{\sigma_{0}} \ln \frac{\tau_{0}}{\sigma_{0}}>\frac{1}{e},
\end{aligned}
$$

then equation (71) is oscillatory by Corollary 8 .

Example 16. Consider the equation

$$
\left(t\left|z^{\prime \prime \prime}(t)\right| z^{\prime \prime \prime}(t)\right)^{\prime}+\left|z^{\prime \prime \prime}(t)\right| z^{\prime \prime \prime}(t)+\frac{q_{0}}{t^{4}}\left|x\left(\frac{t}{2}\right)\right| x\left(\frac{t}{2}\right)=0,
$$

where $t \geq t_{0}=1, z(t)=x(t)+(1 / 3) x(t / 4)$, and $q_{0}>0$. Let $p_{1}$ $=3, p_{2}=2, r(t)=t, \tau(t)=t / 4, \sigma(t)=t / 2, a(t)=1 / 3, p(t)=$ 1 , and $q(t)=q_{0} / t^{4}$. It is easy to check that $\left(H_{1}\right)$ and $\left(H_{3}\right)$ are satisfied. Moreover, we find

$$
\begin{aligned}
& \lim _{t \rightarrow \infty} \inf _{t \rightarrow(t)}^{t}\left(\frac{\mu}{6}\right)^{p_{2}-1} \frac{q(s)(\sigma(s))^{3\left(p_{2}-1\right)} \exp \left(\int_{t_{0}}^{s}(p(\xi) / r(\xi)) d \xi\right)(1-a(\sigma(s)))^{p_{2}-1}}{r^{\left(p_{2}-1\right) /\left(p_{1}-1\right)}(\sigma(s)) \exp \left(\left(\left(p_{2}-1\right) /\left(p_{1}-1\right)\right) \int_{t_{0}}^{\sigma(s)}(p(\xi) / r(\xi)) d \xi\right)} d s \\
& \quad=\lim _{t \rightarrow \infty} \inf _{t / 2}^{t} \frac{\mu}{6} \frac{\left(q_{0} / s^{4}\right)(s / 2)^{3}(2 / 3) s}{(s / 2)^{1 / 2} \exp \left((1 / 2) \int_{1}^{s / 2}(1 / \xi) d \xi\right)} d s=\frac{\mu q_{0}}{36} \ln 2 .
\end{aligned}
$$

Thus, by Corollary 10, all solutions of equation (74) are oscillatory if $q_{0}>36 / \mu e \ln 2$.

Example 17. Consider the equation

$$
\left(t^{2}\left|z^{\prime \prime \prime}(t)\right|^{2} z^{\prime \prime \prime}(t)\right)^{\prime}+t\left|z^{\prime \prime \prime}(t)\right|^{2} z^{\prime \prime \prime}(t)+\frac{q_{0}}{t^{7}}|x(c t)|^{2} x(c t)=0,
$$

where $t \geq t_{0}=1, z(t)=x(t)+a_{0} x\left(\tau_{0} t\right), q_{0}>0$, and $c \in(0,1)$. Let $p_{1}=p_{2}=4, r(t)=t^{2}, \sigma(t)=c t, p(t)=t$, and $q(t)=q_{0} / t^{8}$. It is easy to verify that $\left(H_{1}\right)$ holds. Set $h(s)=s^{6}$, for any $q_{0}$ $>0$; then, it is easy to verify that

$$
\begin{gathered}
\int_{t_{2}}^{\infty}\left(h(s) q(s) \exp \left(\int_{t_{0}}^{s} \frac{p(\xi)}{r(\xi)} d \xi\right) \frac{\sigma(s)^{3\left(p_{1}-1\right)}}{s^{3\left(p_{1}-1\right)}}\right. \\
\left.-\frac{2^{p_{1}-1}}{p_{1}^{p_{1}}} \frac{r(s)\left(h^{\prime}(s)\right)^{p_{1}}}{\left(\varepsilon_{1} s^{2} h(s)\right)^{p_{1}-1} \exp \left(-\int_{t_{0}}^{s}(p(\xi) / r(\xi)) d \xi\right)}\right) d s \\
=\int_{t_{2}}^{\infty}\left(\frac{q_{0} c^{9}}{t_{0}} \frac{1}{s}-\frac{81}{2 \varepsilon_{1}^{3} t_{0}} \frac{1}{s^{2}}\right) d s=\infty,
\end{gathered}
$$

that is, (37) holds. Moreover, one has

$$
\begin{aligned}
\theta(t)= & \lambda^{p_{2}-1 / p_{1}-1} \int_{t}^{\infty}\left(\frac{1}{r(\xi)} \int_{\xi}^{\infty} q(s) \exp \left(\int_{t}^{s} \frac{p(\xi)}{r(\xi)} d \xi\right)\right. \\
& \left.\cdot\left(\frac{\sigma(s)}{s}\right)^{p_{2}-1} d s\right)^{1 /\left(p_{1}-1\right)} d \xi \\
= & \lambda \int_{t}^{\infty}\left(\frac{c^{3}}{\xi^{2}} \int_{\xi}^{\infty} \frac{q_{0}}{s^{7}} \frac{s}{t} d s\right)^{1 / 3} d \xi=\frac{3}{5} \lambda c \sqrt[3]{\frac{q_{0}}{6}} \frac{1}{t^{2}}
\end{aligned}
$$

Thus, by Corollary 14, we obtain that equation (76) is oscillatory if $q_{0}>\max \left\{6(5 / 12 \lambda c)^{3}, 81\left(2 \varepsilon_{1}^{3} c^{9}\right)\right\}$.

\section{Conclusions}

With the help of the comparison strategies involving firstand second-order differential equations and a refinement of the Riccati transformations, some new criteria for oscillation of fourth-order $p$-Laplacian differential equations with middle term are established. The results obtained here complement and extend some known results in [26].

\section{Data Availability}

No data were used to support this study. 


\section{Conflicts of Interest}

The authors declare that there are no conflicts of interest.

\section{Authors' Contributions}

The authors have contributed equally and significantly in this paper. All authors have read and agreed to the published version of the manuscript.

\section{Acknowledgments}

This work is supported by the Natural Science Foundation of China (11571136).

\section{References}

[1] R. P. Agarwal, S. R. Grace, and D. O'Regan, Oscillation theory for difference and functional differential equations, Kluwer Academic Publishers, Dordrecht, The Netherlands, 2000.

[2] R. P. Agarwal, S. R. Grace, and J. V. Manojlovic, "Oscillation criteria for certain fourth order nonlinear functional differential equations," Mathematical and Computer Modelling, vol. 44, no. 1-2, pp. 163-187, 2006.

[3] S. R. Grace, R. P. Agarwal, and J. R. Graef, "Oscillation theorems for fourth order functional differential equations," Journal of Applied Mathematics and Computing, vol. 30, no. 1-2, pp. 75-88, 2009.

[4] E. M. Elabbasy, E. Thandapani, O. Moaaz, and O. Bazighifan, "Oscillation of solutions to fourth-order delay differential equations with middle term," Open Journal of Mathematical Sciences, vol. 3, pp. 191-197, 2019.

[5] O. Moaaz and A. Muhib, "New oscillation criteria for nonlinear delay differential equations of fourth- order," Applied Mathematics and Computation, vol. 377, article 125192, 2020.

[6] C. Cesarano and O. Bazighifan, "Oscillation of fourth-order functional differential equations with distributed delay," Axioms, vol. 8, no. 2, p. 61, 2019.

[7] I. Dassios and O. Bazighifan, "Oscillation conditions for certain fourth-order non-linear neutral differential equation," Symmetry, vol. 12, no. 7, p. 1096, 2020.

[8] O. Moaaz, P. Kumam, and O. Bazighifan, "On the oscillatory behavior of a class of fourth-order nonlinear differential equation," Symmetry, vol. 12, no. 4, p. 524, 2020.

[9] O. Moaaz, I. Dassios, O. Bazighifan, and A. Muhib, "Oscillation theorems for nonlinear differential equations of fourthorder," Mathematics, vol. 8, no. 4, p. 520, 2020.

[10] O. Bazighifan and C. Cesarano, "A philos-type oscillation criteria for fourth-order neutral differential equations," Symmetry, vol. 12 , no. 3 , p. 379, 2020.

[11] N. Parhi and A. Tripathy, "On oscillatory fourth order linear neutral differential equations-I," Mathematica Slovaca, vol. 54, pp. 389-410, 2004.

[12] C. Zhang, T. Li, and S. H. Saker, "Oscillation of fourth-order delay differential equations," Journal of Mathematical Sciences, vol. 201, no. 3, pp. 296-309, 2014.

[13] R. P. Agarwal, O. Bazighifan, and M. A. Ragusa, "Nonlinear neutral delay differential equations of fourth-order: oscillation of solutions," Entropy, vol. 23, no. 2, p. 129, 2021.
[14] O. Bazighifan, "Oscillatory applications of some fourth-order differential equations," Mathematical Methods in the Applied Sciences, vol. 43, no. 17, pp. 10276-10286, 2020.

[15] G. Aronsson and U. Janfalk, "On Hele-Shaw flow of power-law fluids," European Journal of Applied Mathematics, vol. 3, no. 4, pp. 343-366, 1992.

[16] C. Zhang, R. P. Agarwal, and T. Li, "Oscillation and asymptotic behavior of higher-order delay differential equations with $p$-Laplacian like operators," Journal of Mathematical Analysis and Applications, vol. 409, no. 2, pp. 1093-1106, 2014.

[17] T. Li, B. Baculikova, J. Dzurina, and C. Zhang, "Oscillation of fourth order neutral differential equations with $p$-Laplacian like operators," Boundary Value Problems, vol. 56, 58 pages, 2014.

[18] F. Behboudi and A. Razani, "Two weak solutions for a singular $(p, q)$-Laplacian problem," Filomat, vol. 33, no. 11, pp. 33993407, 2019.

[19] C. Cowan and A. Razani, "Singular solutions of a $p$-Laplace equation involving the gradient," Journal of Differential Equations, vol. 269, no. 4, pp. 3914-3942, 2020.

[20] M. Makvand Chaharlang and A. Razani, "A fourth order singular elliptic problem involving $p$-biharmonic operator," Taiwanese Journal of Mathematics, vol. 23, no. 3, pp. 589-599, 2019.

[21] M. Makvand Chaharlang and A. Razani, "Existence of weak solutions for a nonlocal singular elliptic problem," Azerbaijan Journal of Mathematics, vol. 11, no. 1, pp. 80-91, 2021.

[22] M. M. Chaharlang, M. A. Ragusa, and A. Razani, "A sequence of radially symmetric weak solutions for some nonlocal elliptic problem in $\mathbb{R}^{N}$," Mediterranean Journal of Mathematics, vol. 17 , no. 2 , pp. 1-12, 2020.

[23] M. Makvand Chaharlang and A. Razani, "Two weak solutions for some Kirchhoff- type problem with Neumann boundary condition," Georgian Mathematical Journal, vol. 2020, 10 pages, 2020.

[24] S. Liu, Q. Zhang, and Y. Yu, "Oscillation of even-order halflinear functional differential equations with damping," Computers \& Mathematcs with Applications, vol. 61, no. 8, pp. 2191-2196, 2011.

[25] O. Bazighifan and T. Abdeljawad, "Improved approach for studying oscillatory properties of fourth-order advanced differential equations with $p$-Laplacian like operator," Mathematics, vol. 8, no. 5, p. 656, 2020.

[26] O. Bazighifan, "On the oscillation of certain fourth-order differential equations with $p$-Laplacian like operator," Applied Mathematics and Computation, vol. 386, p. 125475, 2020.

[27] O. Moaaz, J. Awrejcewicz, and O. Bazighifan, "A new approach in the study of oscillation criteria of even-order neutral differential equations," Mathematics, vol. 8, no. 2, p. 197, 2020.

[28] O. Moaaz, R. A. el-Nabulsi, O. Bazighifan, and A. Muhib, "New comparison theorems for the even-order neutral delay differential equation," Symmetry, vol. 12, no. 5, p. 764, 2020.

[29] I. T. Kiguradze and T. A. Chanturia, Asymptotic properties of solutions of nonautonomous ordinary differential equations, Kluwer Acad. Publ, Dordrecht, The Netherland, 1993.

[30] G. E. Chatzarakis, S. R. Grace, I. Jadlovská, T. Li, and E. Tunç, "Oscillation criteria for third-order Emden-Fowler differential equations with unbounded neutral coefficients," Complexity, vol. 2019, Article ID 5691758, 7 pages, 2019. 
[31] S. Y. Zhang and Q. R. Wang, "Oscillation of second-order nonlinear neutral dynamic equations on time scales," Applied Mathematics and Computation, vol. 216, no. 10, pp. 28372848, 2010.

[32] B. Baculikova and J. Dzurina, "Oscillation theorems for second-order nonlinear neutral differential equations," Computers \& Mathematcs with Applications, vol. 62, no. 12, pp. 4472-4478, 2011.

[33] C. G. Philos, "On the existence of nonoscillatory solutions tending to zero at $\infty$ for differential equations with positive delays," Archiv der Mathematik, vol. 36, no. 1, pp. 168-178, 1981.

[34] R. P. Agarwal, S. L. Shieh, and C. C. Yeh, "Oscillation criteria for second-order retarded differential equations," Mathematical and Computer Modelling, vol. 26, no. 4, pp. 1-11, 1997. 